\title{
Aicardi syndrome: A report of five Indian cases
}

\author{
T. K. Banerjee, A. Chattopadhyay, A. K. Manglik', B. Ghosh ${ }^{2}$ \\ National Neurosciences Centre, ${ }^{1}$ Shishu Sanjivan and ${ }^{2} B$. R. Singh Hospital, Kolkata, India
}

\begin{abstract}
Five Indian cases of Aicardi syndrome, aged 1-13 years and all with the classic triad of infantile spasm, corpus callosal agenesis and chorioretinal lacunae, were presented. The purpose of this report was to demonstrate the clinical, electroencephalographic (EEG) and radiological spectrum and the treatment outcome of this disorder among the Indians. None of the patient had any family history of similar illness. All had profound psychomotor impairment with no meaningful speech development. There were microphthalmia in 2, optic disc coloboma in 1, interhemispheric cyst in 1, periventricular heterotopia in 2 and thoracolumbar kyphoscoliosis in 2 cases. They all had early onset epilepsy and there were multiple types of seizures. Two cases $(40 \%)$ had the characteristic dissociated burst-suppression pattern in EEG. Two cases whose antiepileptic medications included vigabatrin had complete control of seizure.
\end{abstract}

Key words: Aicardi syndrome, Indian, impaired speech, vigabatrin

Aicardi syndrome (AS), identified first by Jean Aicardi in 1965, is a rare genetic disorder characterized by infantile spasm (IS), corpus callosal agenesis (CCA) and chorioretinal lacunae (CRL). ${ }^{[1,2]}$ This is a X-linked dominant condition and occurs almost exclusively in females, because of early embryonic lethality in hemizygous males. ${ }^{[2]}$ Cerebral malformations like microgyria, heterotopias, intraventricular cysts and choroid plexus papillomas coexist at times. The other occasional findings are abnormalities of ribs and spine, microphthalmia, optic nerve coloboma and asymmetry of cerebral hemispheres.

This is a rare disorder and it has been estimated that there are less than 500 cases worldwide. From India only a few single case reports of AS have been published so far. ${ }^{[3-6]}$ We now report 5 new cases.

Our purpose in this study is to demonstrate the clinical spectrum and outcome of AS cases among the Indians.

We have collected five AS cases in the period between the years 2000-2005. Their diagnosis was based upon the classic triad of IS, CCA and CRL. All the cases had uneventful birth history except that there was a history of maternal oligohydramnios in case No. 5. Case No. 2 had a healthy younger brother, but the rest were the only child of their parents. At the time of birth, their paternal age ranged from 28 to 34 years and maternal age 20 33 years. None had any family history of AS. Their clinical features, investigational findings and outcome of treatment were summarized in Tables 1, 2 and 3. Denver developmental assessment scale was applied to evaluate the psychomotor milestones attained. ${ }^{[7]}$ All had significant psychomotor retardation [Table 1] and none developed any meaningful speech. Case No. 1 aged 13 years was the only one who could make simple communications with nonverbal gestures. Their epileptic seizures required multiple antiepileptic medications (AED); 100\% remission was achieved in case Nos 1 and 5 whose AED included vigabatrin. In case No. 5, seizure stopped completely after vigabatrin was added later to their existing AED [Table 3]. Cerebral

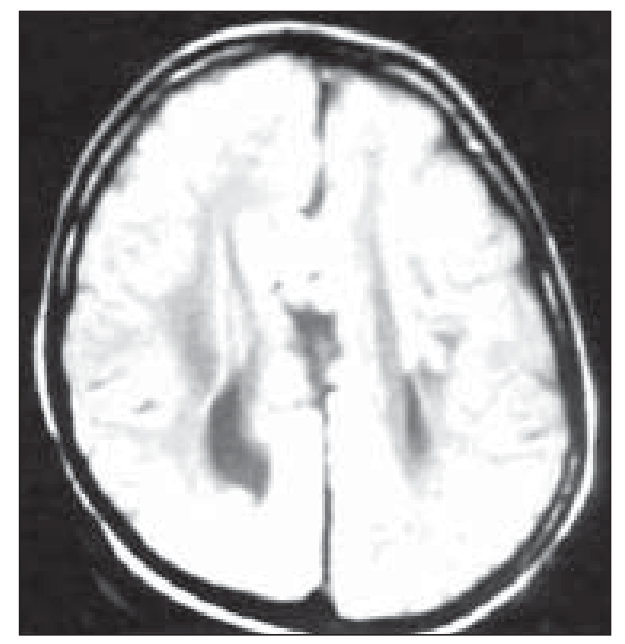

Figure 1: Corpus callosal agenesis and right periventricular heterotopia in cerebral MRI of case No. 1 
Table 1: Salient clinical features of the AS cases

\begin{tabular}{lccccc}
\hline $\begin{array}{l}\text { Case } \\
\text { No. }\end{array}$ & $\begin{array}{c}\text { Age } \\
\text { (years/months) }\end{array}$ & $\begin{array}{c}\text { Seizure onset } \\
\text { (in months) }\end{array}$ & $\begin{array}{c}\text { Seizure } \\
\text { type }\end{array}$ & Eye findings & $\begin{array}{c}\text { Maximum developmental } \\
\text { age attained (in months) }\end{array}$ \\
1 & 13 & 3 & IS & CRL & 14 \\
2 & 4 & 3 & IS, T, C,adv & CRL, left microphthalmia & 5 \\
3 & 3 & 4 & IS, T,C, myoclonus & CRL, right micropthalmia & 5 \\
4 & 1 & 4 & IS & CRL & 1 \\
5 & 1 year 2 months & 1.5 & IS, C & CD coloboma & 1 \\
\hline
\end{tabular}

IS, infantile spasm; C, clonic seizure; T, tonic seizure; CRL, chorioretinal lacunae; adv, adversive seizure; OD, optic disc.

\begin{tabular}{llll}
\hline & & Table 2: Investigational findings of the AS cases \\
\hline Case No. & X-ray spine & EEG & Cerebral MRI \\
1 & TLKS & Right focal seizure & CCA, periventricular heterotopia \\
2 & Normal & Multifocal spikes & CCA, interhemispheric cyst \\
3 & TLKS, spina bifida occulta & DBS & CCA, subcortical and periventricular heterotopias \\
4 & Normal & Focal spikes & CCA \\
5 & Normal & DBS & CCA \\
\hline
\end{tabular}

TLKS, thoracolumbar kyphoscoliosis; DBS, dissociated burst-suppression pattern; CCA, corpus callosal agenesis.

\begin{tabular}{lll}
\hline & \multicolumn{2}{c}{ Table 3: Treatment outcome in AS cases } \\
\hline Case & Antiepileptics used & $\%$ improvement in seizure \\
1 & VGA, VPA, CLO & $100 \%$ \\
2 & VGA, VPA & No change, noncompliant \\
3 & VPA, CLOB & $75 \%$ \\
4 & VPA & Lost to follow-up \\
5 & ACTH, CLO, VPA & $50-75 \%$ \\
& Later above + VGA & $100 \%$ \\
\hline
\end{tabular}

$\overline{\text { VGA, vigabatrin; VPA, valproate; CLO, clonazepam; CLOB, clobazam; PHE, }}$ phenobarbitone; $\mathrm{ACTH}$, adreno cortico trophic hormone; $\mathrm{CBZ}$, carbamazepine.

magnetic resonance imaging (MRI) finding and funduscopic picture of AS patients were illustrated [Figures 1, 2 and 3].

Case No. 4 was lost to follow up and case No. 5 died of aspiration pneumonia. Case Nos 1, 2 and 3 were being followed up for the last 3 years. Follow-up electroencephalography (EEG) showed partial improvement in epileptiform activities with improvement in seizure frequency but dissociated burst-suppression pattern persisted. Flash visual evoked potential was performed in two cases; the result was normal in case 1 and bilaterally absent in case 2 , both before and after vigabatrin intake.

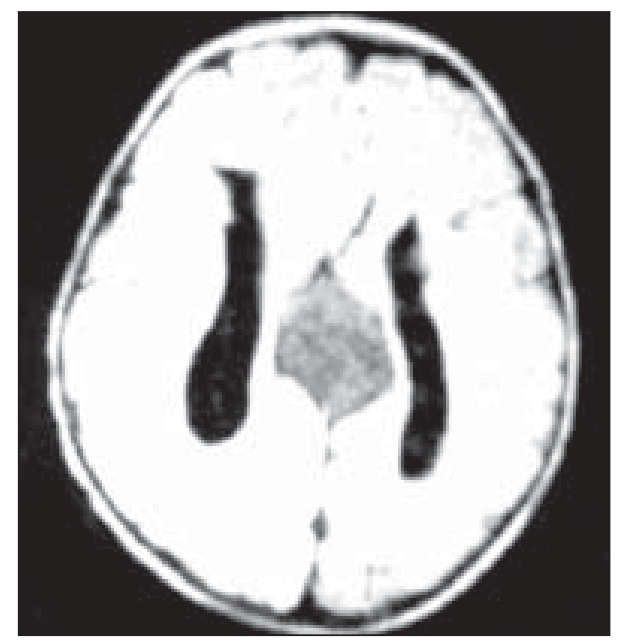

Figure 2: Corpus callosal agenesis with interhemispheric cyst in cerebral MRI of case No. 2

\section{Discussion}

The diagnosis of AS is based upon the classic triad of CCA, CRL and IS. But there is a range of costovertebral, ocular and cerebral abnormalities associated with this disorder. ${ }^{[2]}$ The cerebral gray-matter heterotopias and other cortical malformations act as epileptogenic foci. ${ }^{[2]}$ Their seizures typically start in early childhood and are usually intractable. Besides IS, other seizure types are also demonstrated. Dissociated burst-suppression or burstsuppression pattern appearing asymmetrically in either cerebral hemisphere is a characteristic EEG finding in AS. ${ }^{[8]}$ The developmental delay in AS is generally profound, involving both motor and language skills. Chevrie and Aicardi in their analysis of 184 patients of AS observed that none had acquired meaningful speech. ${ }^{[9]}$ But of late a larger spectrum of the disease has been recognized and it had been found that higher functioning AS individuals do exist. ${ }^{[2]}$ Most of the AS cases die at an early age primarily due to aspiration pneumonitis. But some do live into their adolescent years and even in their twenties. ${ }^{[2]}$ Good visual function in AS patients do occur if the fovea is uninvolved with CRL, as was in case No. 1 in our series.

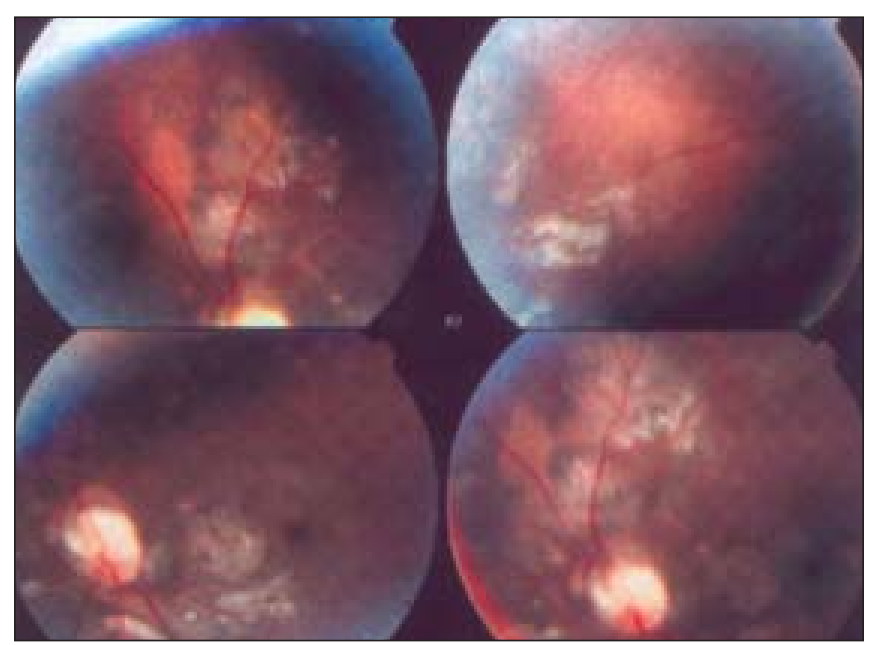

Figure 3: Funduscopic picture of case No 3 showing chrioretinal lacunae 


\section{Banerjee et al: Aicardi syndrome}

Cerebral heterotopias, interhemispheric cysts, optic nerve coloboma, microphthalmia, thoracolumbar kyphoscoliosis present in our patients are the known associated features in AS. Severe psychomotor impairment and absence of meaningful speech noted among our patients had also been noted among the earlier reported Indian cases of AS. ${ }^{[3]-[6]}$ The seizures were of early onset, intractable and there were multiple seizure types. The two patients in our study who were completely seizure-free were the ones, whose AED included vigabatrin. A recent report observed similar good results with vigabatrin and recommends this as the first-line drug in the treatment of seizure in AS cases. ${ }^{[10]}$

Aicardi syndrome is indeed a rare neurological illness. But as observed from our study and from the earlier case reports, ${ }^{[3-6]}$ it does exist in our country. Therefore, any female child with recurrent seizure occurring in early childhood should be investigated appropriately to exclude AS. Vigabatrin may be considered among the first-line AED; however, one should keep in mind the risk of retinal toxicity with this medication.

\section{References}

1. Aicardi J, Lefebvre J, Lerique Koechlin A. A new syndrome: Spasms in flexion, callosal agenesis, ocular abnormalities. Electroencephalogr Clin Neurophysiol 1965;19:609-10.

2. Rosser TL, Acosta MT, Packer RJ . Aicardi Syndrome:Spectrum of Disease and Long-Term Prognosis in 77 Females. Pediatr Neurol 2002;27:343-6.

3. Puri V, Gupta BK, Parihar PS, Anand I. The Aicardi Syndrome. Indian Pediatr 1991;28:1074-7.

4. Singhi PD, Gupta A, Agarwal A. Aicardi Syndrome. Indian Pediatr 1991;28:1513-6.

5. Shah KN, Rajadhyaksha S, Shah VS, Wakde M. EEG recognition of holoprosencephaly and Aicardi Syndrome. Indian J Pediatr 1992;59:103-8.

6. Wadia RS, Joshi A, Vaidija SD, Manavi AB, Kelker. The Aicardi Syndrome. Neurol India 1985;33:149-54.

7. Frankenburg WE, Dodds JB. The Denver Developmental Assessment (Denver II). Denver: University of Colorado Medical School; 1990.

8. Ohtsuka Y, Oka E, Terasaki T, Ohtahara S. Aicardi svndrome: a longitudinal clinical and electroencephalographic study. Epilepsia 1993;34:627-34.

9. Chevrie J.J, Aicardi J. The Aicardi syndrome. In: Pedley TA, Meldrum BS, eds Recent advances in epilepsv, vol. 3. Edinburgh: Churchill Livingstone; 1986. p. 189-210.

10. Chau V, Karvelas G, Jacob P, Carmant L. Early treatment of Aicardi syndrome with vigabatrin can improve outcome. Neurology 2004;63:1756-7.

Accepted on 09-08-2005 